\title{
CONSUMO DE MEDICAMENTOS EM REGIÃO DO ESTADO DE SÃO PAULO (BRASIL), $1985^{*}$
}

\author{
Maria Jacira S. Simóes** \\ Adalberto Farache Filho**
}

\begin{abstract}
SIMÕES, M.J.S. \& FARACHE FILHO, A. Consumo de medicamentos em região do Estado de São Paulo (Brasil), 1985. Rev. Saúde públ., S. Paulo, 22:494-9, 1988.

RESUMO: Com o objetivo de avaliar as características do consumo de medicamentos na populaçăo urbana de Araraquara, SP, Brasil, foram coletados dados, por meio de entrevistas domiciliares, de uma amostra da populaçáo que consumiu pelo menos um medicamento nos quinze dias que antecederam a data da entrevista. O estudo foi realizado no periodo de agosto a setembro de 1985 . Verificou-se que $42,1 \%$ dos medicamentos utilizados foram adquiridos sem prescriçăo médica. $O$ consumo entre o sexo feminino foi maior que para o sexo masculino. Na automedicaçăo o grupo que apresentou taxa mais elevada, segundo a faixa etária, foi o de 50 anos e mais, com $31,6 \%$. Grande parte do consumo de medicamentos constituiu-se dos industrializados $(\mathbf{9 7 , 6 \% )}$. As prescriçoes médicas, feitas em consultas anteriores, e avaliadas como bem sucedidas foram retómadas em situaçōes diversas $(12,0 \%)$, revelando o importante papel que o médico desempenha na formação dos critérios de escolha dos remédios utilizados nas práticas de automedicação. O farmacêutico e/ou balconista de farmácia contribui com $10,0 \%$ dos medicamentos usados que tiveram essa via de indicação. As orientaçoes feitas por amigos, vizinhos e parentes $(9,1 \%)$ revelaram intenso circuito de trocas de socializaçoes quanto aos quadros mobidos e indicaçōes terapéuticas.
\end{abstract} mentos.

UNITERMOS: Uso de medicamentos. Hábitos de consumo de medicamentos. Prescriçăo de medica-

\section{INTRODUÇÃO}

A automedicação, bem como o crescente e, muitas vezes, abusivo, errôneo e indiscriminado número de prescriçōes, está ocorrendo mundialmente ${ }^{11,14,22}$.

Como nenhum fármaco é totalmente inócuo ao organismo, a incidência de efeitos indesejáveis aumenta a cada dia, levando à iatrogenia medicamentosa, causa de elevado número de hospitalização" ${ }^{11}$.

Segundo Cunha ${ }^{8}$, em 1980 o consumo médio de medicamentos pela população brasileira apresentava evolução superior a $50 \%$ em relação a 1974.

Os dados dos relatórios da Abifarma' de $1977 / 78$ e 1978/79 confirmam que estavam registrados no Ministério da Saúde 3.496 medicamentos originais e 3.596 similares, num total de 7.065. Considerando que um mesmo medicamento pode ter mais de uma forma farmacêutica, esses 7.065 medicamentos somavam o total de 11.300 apresentaçôes.

No Brasil não existem dados sobre os gastos em pesquisas e desenvolvimento de produtos éticos para o uso humano. Sabemos pelos dados sanitários que a verminose é problema prio- ritário de saúde no Brasil, sendo de se esperar que a venda de vermífugos estivesse entre os medicamentos mais consumidos no país; entretanto, afirmam os dados da Abifarma' fornecidos a CPI da Indústria Farmacêutica, em 1979, que a venda de vermífugos correspondia apenas a 1,3\% do total de venda de medicamentos.

Comentam alguns estudiosos do assunto $0^{8,11,22}$ que, se o Brasil não despertar para a necessidade da adoção de um conjunto de ações urgentes, estaremos condenados irremediavelmente ao domínio externo de nossa condição de vida. Cada vez mais os medicamentos se afastarão da possibilidade de nacionalização, cacia vez mais seremos tratados com princípios ativos desenvolvidos em outros países, para outros povos com características ambientais de vida completamente diferente da nossa.

Em 1977, a Örganização Mundial de Saúde ${ }^{21}$ propôs-se a fazer a primeira linha de seleção de medicamentos essenciais, os quais dependerão das necessidades de saúde, estrutura e grau de desenvolvimento dos serviços de saúde de cada país e dos fatores como: grau de prevalência de determinadas enfermidades, meios disponíveis para aplicação do tratamento, capacidade e exi periência do pessoal disponivel.

- Pesquisa financiada pela Fundação de Amparo d Pesquisa do Estado de Săo Paulo (FAPESP). Proc. Biomedicina n: 84/2459-6.

* Departamento de Ciências Biológicas da Faculdade de Ciências Farmacêuticas de Araraquara da Universidade Estadual Paulista "Júlio de Mesquita Filho" - Rua Expedicionários do Brasil, 1621 - 14800 - Araraquara, SP - Brasil 
No Brasil, a partir de 1980 , tem-se tentado mudar a política de medicamentos pelas medidas adotadas pela Divisão Nacional de Vigilância Sanitária de Medicamentos ${ }^{23}$. Dentre as principais medidas que de imediato foram adotadas, temos a rígida restrição do licenciamento de associaçōes medicamentosas e a implantação de normas que cobrem as principais necessidades do Brasil na área de medicamentos como formulário de cadastro de produtos, nomes genéricos de substâncias farmacologicamente ativas, bula padrão, entre outros.

No nosso meio, assim como em qualquer país subdesenvolvido, a indústria farmacêutica é como qualquer outra indústria, de propósito mercantilista, dirigida para o lucro privado e só, secundariamente, como sub-produto, voltada para uma das mais dramáticas necessidades do homem: a superação da doença ${ }^{24}$.

O caráter mercantil dessa indústria faz com que ela, na busca de eficiência, antes que a eficácia social, adote quase todas as estratégias e procedimentos da indústria em geral: economia de escala, padronização de produtos, massificação de consumo, e outras. Não temos uma indústria farmacêutica $d o$ Brasil, mas tão somente uma indústria farmacêutica no Brasil. Pois mais de $80 \%$ do faturamento das empresas do setor, em operação no país, corresponde às atividades das transnacionais $s^{8,12,13}$.

Há que se procurar soluções para os problemas da assistência farmacêutica com que nos defrontamos e para cuja solução não podemos prescindir da colaboração de todos. Assim, o presente trabalho tem por objetivo conhecer algumas características do consumo de medicamentos da população de Araraquara, como início de estudo mais amplo.

\section{MATERIAL E MÉTODOS}

População de análise - Foi constituída da totalidade dos residentes da zona urbana do Município de Araraquara que nos últimos 15 dias (em relação à data da entrevista) consumiram pelo menos um medicamento.

Procedimento e amostragem - Para definição da população estudada, foram selecionados os domicílios por meio de amostragem por conglomerado, em três etapas:

1.) Unidade primária de amostragem (UPA): setor censitário (ou conjunto de setores censitários)

2.) Unidade secundária de amostragem (USA): quarteirão (ou conjunto de quarteirões)

3.) Unidade de terceira etapa: domicílio.
Assim, todas as pessoas residentes nos municípios selecionados e que consumiram pelo menos um medicamento no período fixado constituiram a população de estudo. Os indivíduos foram distribuídos segundo os grupos etários: $<5 ; 5-19 ; 20-49 ; 50$ anos e mais.

Os dados foram coletados por meio de entrevistas domiciliares, no período de agosto a setembro de 1985.

Foram selecionados 551 domicílios com uma população de 2.150 , na qual foram encontrados 853 indivíduos que consumiram medicamentos; destes 27 não souberam informar o nome do medicamento utilizado.

As informações coletadas e analisadas foram:

- Quanto à identificação pessoal - nome, idade e sexo.

- Quanto ao medicamento: nome comercial, sintoma ou condição para os quais foram usados, pessoa que orientou o uso.

\section{RESULTADOS E DISCUSSÃO}

Na Tabela 1 mostramos a distribuição da população estudada segundo o sexo, idade e consumo de medicamentos com e sem prescrição médica. Com esses resultados calculamos as prevalências em cada grupo segundo dados da Tabela 2. Observamos que quanto ao uso de medicamentos com prescrição, segundo o sexo, há maior percentagem para 0 sexo feminino que o masculino, $23,5 \%$ e $16,5 \%$, respectivamente.

Por grupo de idade e uso de medicamentos com prescrição, a menor percentagem é no grupo de 20 a 49 anos $(8,9 \%)$ para o sexo masculino e de 5 a 19 anos $(14,7 \%$ ) para o feminino.

No uso de medicamentos sem prescrição, e por sexo, não encontramos diferença quando observados os totais; quando associamos com a idade verificamos que no grupo de 20 a 49 anos, para o sexo masculino, o consumo é bem menor em relação aos demais grupos de idade $(8,1 \%)$. Enquanto que no sexo feminino só houve grande variação de percentagem em relação aos demais grupos etários para o grupo de 50 anos e mais $(30,2 \%)$ ) (Tabela 2$)$.

No grupo etário de 20 a 49 anos encontramos uma grande diferença por sexo quanto ao consumo de medicamento. Com prescrição, a percentagem foi $8,9 \%$ para o sexo masculino e $16,7 \%$ para o feminino, naqueles que consumiram sem prescrição foi de $8,1 \%$ e $20,8 \%$, respectivamente (Tabela 2).

Esta variação quanto ao sexo e idade foram 


\section{TABELA 1}

Distribuiçz̃o da população amostrada, segundo a idade, sexo e uso de medicamento com e sem prescrição médica. Araraquara, SP, 1985 .

\begin{tabular}{|c|c|c|c|c|c|c|c|c|c|c|}
\hline \multirow{2}{*}{ Idade } & \multirow[b]{2}{*}{ Sexo } & \multicolumn{3}{|c|}{ Com prescrição } & \multicolumn{3}{|c|}{ Sem preșcrição } & \multicolumn{3}{|c|}{ População residente } \\
\hline & & M & F & $T$ & $\mathbf{M}$ & $\mathbf{F}$ & $\mathrm{T}$ & $\mathbf{M}$ & $\mathrm{F}$ & $\mathbf{T}$ \\
\hline $\begin{array}{r}5 \\
5.19 \\
20.49 \\
50 \text { e mais }\end{array}$ & & $\begin{array}{l}39 \\
39 \\
44 \\
48\end{array}$ & $\begin{array}{r}25 \\
44 \\
88 \\
100\end{array}$ & $\begin{array}{r}64 \\
83 \\
132 \\
148\end{array}$ & $\begin{array}{l}25 \\
58 \\
40 \\
49\end{array}$ & $\begin{array}{r}25 \\
62 \\
110 \\
52\end{array}$ & $\begin{array}{r}50 \\
120 \\
150 \\
101\end{array}$ & $\begin{array}{l}118 \\
280 \\
495 \\
148\end{array}$ & $\begin{array}{l}109 \\
300 \\
528 \\
172\end{array}$ & $\begin{array}{r}227 \\
580 \\
1023 \\
320\end{array}$ \\
\hline Total & & $172^{*}$ & $261^{*}$ & $427 *$ & 172 & $250^{* *}$ & $421 * *$ & 1041 & 1109 & 2150 \\
\hline
\end{tabular}

* Excluídos 2 indivíduos do sexo masculino e 2 do feminino por falta de informação quanto à idade.

** Excluído um do sexo feminino por falta de informação quanto à idade.

TABELA 2

Distribuição do percentual da prevalência do consumo de medicamento com e sem prescrição médica, segundo a idade e sexo. Araraquara, $S_{9}, 1985$.

\begin{tabular}{|c|c|c|c|c|c|c|c|}
\hline \multirow[t]{2}{*}{ Idade } & & \multicolumn{3}{|c|}{ Com prescrição } & \multicolumn{3}{|c|}{ Sem prescrição } \\
\hline & Sexo & $\underset{\%}{\mathrm{M}}$ & $\begin{array}{l}\mathrm{F} \\
\%\end{array}$ & $\begin{array}{l}\mathrm{T} \\
\%\end{array}$ & $\underset{\%}{\mathbf{M}}$ & $\begin{array}{l}\mathrm{F} \\
\%\end{array}$ & $\underset{\%}{\mathbf{T}}$ \\
\hline $\begin{array}{r}5 \\
5 \text { a } 9 \\
20 \text { a } 49 \\
50 \text { e mais }\end{array}$ & & $\begin{array}{r}33,0 \\
13,9 \\
8,9 \\
32,4\end{array}$ & $\begin{array}{l}22,9 \\
14,7 \\
16,7 \\
58,1\end{array}$ & $\begin{array}{l}28,2 \\
14,3 \\
12,9 \\
46,2\end{array}$ & $\begin{array}{r}21,2 \\
20,7 \\
8,1 \\
33,1\end{array}$ & $\begin{array}{l}22,9 \\
20,7 \\
20,8 \\
30,2\end{array}$ & $\begin{array}{l}22,0 \\
20,7 \\
14,7 \\
21,5\end{array}$ \\
\hline Total & & 16,5 & 23,5 & 19,9 & 22,5 & 22,5 & 19,6 \\
\hline
\end{tabular}

também evidenciadas em outros trabalhos realizados $2,6,20,25$.

Segundo estudo realizado por Dean ${ }^{9}$ (1981), a maior medicalização da mulher é observada de maneira generalizada no ocidente e se deve à intensa intervenção médica sobre as condiçōes fisiologicas femininas, que se acentua com a extensão de programas preventivos.

Quando estudamos a automedicação e a prescrição médica, observamos, segundo dados da Tabela 3, que 58,0\% dos medicamentos são prescritos por médico.

Em Nova Iguaçu ${ }^{16}, 48,4 \%$ dos medicamentos utilizados nos 15 dias que antecederam à pesquisa eram não prescritos. Vemos que em Araraquara esta taxa é mais elevada. Inclusive quando comparamos com Ribeirão Preto ${ }^{2}$, onde a taxa encontrada de medicamentos adquiridos mediante receita médica, entre os utilizados no período do estudo, foi de $30,8 \%$.

Esta alta taxa encontrada no nosso meio aponta o papel das instituições médicas e do médico promovendo a elevação do consumo de drogas.
TABELA 3

Distribuição dos medicamentos consumidos no período de estudo, segundo fonte de orientação. Araraquara, SP, 1985.

\begin{tabular}{lcc}
\hline \multicolumn{1}{c}{ Fonte de orientação } & $\begin{array}{c}\text { Medicamentos consumidos } \\
\text { Nọ }\end{array}$ \\
\hline Prescrição médica & 928 & 57,0 \\
Médico em consultas & 192 & 12,0 \\
anteriores & 146 & 9,1 \\
Parente, vizinho, amigo & 2 & 0,1 \\
Médium, benzedor & 160 & 10,0 \\
Farmacêutico e/ou empre- & 3 & 0,2 \\
gado de Farmácia & 172 & 10,7 \\
Propaganda (rádio ou TV) & $1.603 *$ & 100,00 \\
A própria pessoa & & \\
\hline Total & &
\end{tabular}

* O medicamento consumido pode ter mais de uma orientaçâo. 
A segunda taxa mais elevada de consumo de medicamento em Araraquara foi de $12,0 \%$ que, segundo os entrevistados, é resultado de uma prescrição médica anterior, feita para o mesmo problema.

Em Ribeirão Preto ${ }^{2}$ esta taxa corresponde a $24 \%$, o que nos leva a constatar o papel do médico na difusão de marcas de medicamentos, na légitimação do uso de fármacos, orientando as práticas de autoprescrição.

O gasto bastante elevado com propaganda efetuado pela indústria farmacêutica, e cujo principal alvo é o médico, tem, portanto efeito que extrapola a magnitude das vendas de medicamentos prescritos $7,12,13,15,18,26$.

Menendez ${ }^{19}$ também assinala a figura "pedagógica" do médico nas condutas terapêuticas, atribuindo-lhe peso na indução à automedicação.

O farmacêutico, na maioria dos casos balconistas da drogaria ou farmácia, foi apontado como orientador do uso de $10,04 \%$ dos medicamentos consumidos pela amostra pesquisada.

Valores semelhantes foram observados por Barros $^{2}$ que encontrou $12,9 \%$ em Ribeirão Preto-SP e Cabral de Barros 3 , que encontrou nas farmácias domiciliares, de uma amostra da clientela de previdenciários de Recife-PE, 9,7\% de medicamentos comprados sob prescrição de farmacêutico, e por Cordeiro' que constatoụ em pesquisa realizada em quatro grupos sociais, 0 uso de $9,09 \%$ de medicamentos orientados por farmacêuticos entre os utilizados pelos "gerentes" e 13,9\% entre os consumidos por operários da construção civil.

Quanto aos meios de comunicação de massa, não foi encontrada em Araraquara influência importante no consumo de remédios $(0,2 \%)$.

Barros $^{2}$, em Ribeirão Preto, observou influência relativamente pequena, $1,1 \%$ e Cabral de Barros ${ }^{3}$ em Recife encontrou apenas 2,6\% de remédios adquiridos pelo efeito de propaganda.

Em Araraquara uma procura importante de automedicação $(19,8 \%)$ foi atribuída à orientação de pessoas do relacionamento do paciente $(9,1 \%)$ ou a uma escolha pessoal $(10,7 \%)$. Estas constituem, na realidade, fontes secundárias, ficando encoberta a fonte original que gerou o conhecimento e influenciou sobre a seleção do remédio (médico, farmacêutico ou meio de comunicação de massa).

A automedicação é prática comum também nos países desenvolvidos, que cotam com amplos recursos médicos, e naqueles com sistema de saúde cobrindo toda a população. A questão é caracterizar o tipo de medicação e em que contexto se dá. Nesses países, a automedicação, em geral, é por problemas mais simples e segue as normas da medicina científica ${ }^{5}$.

Em Araraquara, segundo dados da Tabela 4, $7,39 \%$ das pessoas que consumiram medicamentos usaram 4 ou mais remédios no período de estudo. Dados semelhantes também foram recolhidos em Ribeirão Preto ${ }^{2,10}, 7,3 \%$.

Segundo dados da Tabela 5, observamos que as pessoas de mais idade usam medicamentos com frequiência e em números elevados, o que deve expô-las a grande incidência de reações adversas. Fato também comentado em alguns trabalhos onde os autores Barros ${ }^{2}$ e Caranasos 4 observaram, em pesquisas sobre reaçס̄es adversas, o aumento de sua ocorrência com a idade.

A frequêencia dos analgésicos e antitérmicos $(21,6 \%)$ foi bem elevada no nosso meio. Foi o grupo de medicamentos mais consumido. Quanto ao uso de antibióticos e quimioterápicos foi de $12,2 \%$.

Comenta Pacheco ${ }^{22}$ que embora o consumo seja muito variável entre as diferentes localidades, a forma inadequada e desnecessária com que os antibióticos são prescritos tem sido freqüentemente relatada, assim como apontados os riscos decorrentes dessa prática.

A elevada proporção de remédios com ação terapêutica sobre o aparelho cardiovascular, $17,2 \%$, em Araraquara, nos faz supor um aumento das doenças degenerativas. Pois, a medida que aumenta a esperança de vida de uma população e que avança o processo de industrialização e urbanização, cresce 0 aumento das doenças degenerativas.

\section{TABELA 4}

Distribuição dos indivíduos segundo o número de remédios utilizados. Araraquara, SP, 1985.

\begin{tabular}{lcc}
\hline $\begin{array}{l}\text { Número de } \\
\text { Medicamentos }\end{array}$ & População \\
Usados & 413 & $\%$ \\
\hline 1 medicamento & 230 & 50,1 \\
2 medicamentos & 121 & 27,8 \\
3 medicamentos & 61 & 14,7 \\
4 e mais & 825 & 7,4 \\
\hline Total & 100,00 \\
\hline
\end{tabular}

O consumo das drogas que atuam no sistema cardiovascular é maior na faixa etária acima de 50 anos $(38,1 \%)$. O contrário sucede com o uso de drogas com ação sobre o aparelho respiratório, que declina com o aumento de idade. 
TABELA 5

Tipos de medicamentos utilizados, segundo o grupo etário do consumidor. Araraquara, SP, 1985.

\begin{tabular}{|c|c|c|c|c|c|c|c|c|c|c|}
\hline \multirow{2}{*}{ Grupos de Medicamentos } & \multicolumn{2}{|c|}{$<5$ anos } & \multicolumn{2}{|c|}{5.19 anos } & \multicolumn{2}{|c|}{20.49 anos } & \multicolumn{2}{|c|}{$50 e+$} & \multicolumn{2}{|c|}{ Total } \\
\hline & No & $\%$ & Nọ & $\%$ & No & $\%$ & No? & $\%$ & No & $\%$ \\
\hline Vitaminas $\bullet$ antianemicos & 22 & 12,9 & 38 & 12,5 & 29 & 6,4 & 21 & 4,2 & 110 & 7,7 \\
\hline $\begin{array}{l}\text { Antibióticos e quimiote- } \\
\text { rápicos }\end{array}$ & 32 & 18,8 & 52 & 17,0 & 55 & 12,1 & 35 & 7,1 & 174 & 12,2 \\
\hline $\begin{array}{l}\text { Analgésicos e antitérmi- } \\
\text { cos }\end{array}$ & 44 & 25,9 & 90 & 29,5 & 104 & 23,0 & 69 & 13,9 & 307 & 21,6 \\
\hline $\begin{array}{l}\text { Ação terapêutica apare- } \\
\text { lho respiratório }\end{array}$ & 53 & 31,2 & 87 & 28,5 & 56 & 12,4 & 36 & 7,3 & 232 & 16,3 \\
\hline Antiespasmódicos & 3 & 1,8 & 2 & 0,6 & 29 & 6,4 & 24 & 4,8 & 58 & 4,1 \\
\hline $\begin{array}{l}\text { Ação terapeutica apare- } \\
\text { lho digestivo }\end{array}$ & 7 & 4,1 & 6 & 2,0 & 39 & 8,6 & 34 & 6,9 & 86 & 6,0 \\
\hline $\begin{array}{l}\text { Ação sob o Sistema } \\
\text { Nervoso Central }\end{array}$ & - & - & 10 & 3,3 & 46 & 10,1 & 45 & 9,4 & 101 & 7,1 \\
\hline $\begin{array}{l}\text { Ação terapêutica apare- } \\
\text { lho cardiovascular }\end{array}$ & - & - & 1 & 0,3 & 51 & 11,3 & 193 & 39,0 & 245 & 17,2 \\
\hline Verminoses & 7 & 4,1 & 9 & 2,9 & 1 & 0,2 & 1 & 0,2 & 18 & 1,3 \\
\hline Outros medicamentos & 2 & 1,2 & 10 & 3,3 & 43 & 9,5 & 37 & 7,7 & 92 & 6,5 \\
\hline Total & 170 & 100,00 & 305 & 100,00 & 453 & 100,00 & 495 & 100,00 & 1423 & 100,00 \\
\hline
\end{tabular}

Obs. : 112 medicamentos não foram classificados porque eram homeopáticos ou fitoterápicos.

\section{CONCLUSÃO}

1. Verificou-se que a percentagem do consumo de medicamento sem prescrição médica foi bem elevada $(42,1 \%)$.

2. Quanto ao sexo, a automedicação entre as mulheres no grupo etário de 20 a 49 anos foi mais que o dobro em relação aos homens $(20,8 \%$ e $8,1 \%$, respectivamente).

3. Quanto ao tipo de orientação, $12,0 \%$ dos medicamentos consumidos foi resultado de uma prescrição médica anterior. O farmacêutico e/ou balconista de farmácia contribuiu com 10,0\%. A automedicação atribuída à orientação de pessoas do seu relacionamento contribuiu com $9,1 \%$ e à própria escolha com $10,7 \%$. A propaganda de massa não influenciou no consumo, pois a taxa en- contrada foi de apenas $0,2 \%$.

4. Quanto ao número de medicamentos usados, $50,1 \%$ usou um remédio; $27,8 \%$ dois remédios; $14,7 \%$ três remédios e $7,4 \%$ consumiu quatro ou mais remédios durante 0 período de estudo.

5. Os grupos de medicamentos mais consumidos segundo o valor decrescente foram: analgésicos e antitérmicos $-21,6 \%$; ação terapêutica sobre o aparelho cardiovascular - 17,2\%; ação terapêutica sobre o aparelho respiratório - 16,3\%; antibióticos e quimioterápicos - 12,2\%; vitaminas e antianêmicos - 7,7\%; ação sob o sistema nervoso central $-7,1 \%$; ação terapêutica sobre 0 . aparelho digestivo $-0,04 \%$; antiespasmodico $-4,1$; verminose $-1,3 \%$ e demais medicamentos $-6,5 \%$. 
SIMŐES, M.J.S. \& FARACHE FILHO, A. [Consumption of Medicines in region of S. Paulo State (Brazil), 1985] Rev. Saúde públ., S. Paulo, 22:494-9, 1988.

ABSTRACT: The objective of this study who the evaluation of the characteristics of the consumption of medicines by the urban population of Araraquara, S. Paulo, Brazil. The data were collected by means of house to house interviews during August and September, 1985. It was discovered that $42.1 \%$ of medicines used had been acquired without medical prescriptions. The consumption of medicines taken by the fermale population was larger than taken by the male. In terms of self-medication the highest rate of consumption was found in the age group of 50 and above $(31.6 \%)$. Industrialized medicines acounted for the greater part of the consumption $(97.6 \%)$. Medical prescriptions which had been received on previous visits to doctors and were taken in different situations $(12.0 \%)$, which shows the importance of the doctor's role and criteria as regards medicines used for self-medication. The person of the pharmacist and /or drug store resistant contributes with $10.0 \%$ of the medicines prescribed and taken in this way. Suggestions made by friends, relations and neighbors $(9.1 \%)$ reveal the intense exchange of remedies in relation to morbid states and therapeutical indications.

UNITERMS: Drug utilization. Drug use habits. Prescriptions, drug.

\section{REFERÊNCIAS BIBLIOGRÁFICAS}

1. ASSOCIAÇÃO BRASILEIRA DE INDÚSTRIA FARMACÊUTICA. Perfil da indústria farmacêutica no Brasil. Rio de Janeiro, 1979.

2. BARROS, M.B. de A. Saúde e classe social um estudo sobre morbidade e consumo de medicamentos. Ribeirão Preto, 1985. [Tese de Doutoramento - Faculdade de Medicina de Ribeirão Preto da USP].

3. CABRAL DE BARROS, J.A. A medicalização da clientela previdenciária. São Paulo, 1982. [Dissertação de Mestrado - Faculdade de Medicina da USP].

4. CARANASOS, G.L.; SEWART, R.B.; CLUFF, L.E. Drug induced illness leading to hospitalization. $J$. Amer. med. Ass., 228:713-7, 1974.

5. CARLSEN, H.; CRISTENSEN, F.; HOLST, E. Drug consumption in Denmark. Acta soc. med. scand., 1:59-63, 1971.

6. CHOI, J.W. Out-of-pocket cost and acquisition of prescribed medicines: United States, 1973. Vital Hith Statist. Sér. 10, (108) 1977.

7. CORDEIRO, H. A indústria da saúde no Brasil. Rio de Janeiro, Graal/CEBES, 1980.

8. CUNHA, B.C. de A. Medicamentos: fator de saúde? São Paulo Editora Assessoria Jornalistica, 1981.

9. DEAN, K. Self-care response illness: a selected review. Soc, Sci. Med., 15A:673-87, 1981.

10. DUNNEL \& CARTWRIGHT apud BARROS².

11. GARRAFA, V. Contra o monopólio da da saúde: temas para debate. Rio de Janeiro, Editora Achiami, 1983.

12. GIOVANNI, G. A questão dos remédios no Brasil (produção e consumo). São Paulo, Ed. Polis, 1980.

13. LANDMANN, J. Evitando a saúde e promovendo a doença - o sistema de saúde no Brasil. $2^{2}$ ed. Rio de Janeiro, Ed. Achimé, 1982.

14. LAPPORTE, J.R.; PORTA, M.; CAPELLA, D.; ARVAV, J. Drugs in the Spanish health system. Int. J. Hlth Serv., 14:635-48, 1984.

15. LEFEVRE, F. A função simbólica dos medicamentos. Rev. Saúde públ., S. Paulo, 17:500-3, 1983.

16. LOYOLLA, M.A.R. Medicina popular ou sistema paralelo de saude; relatório de pesquisa. Rio de Janeiro, 1978. [mimeografado].
17. MANUAL de classificação, estatística internacional de doenças, lesões e causas de dbitos; 9? revisão, 1975. São Paulo, Centro da OMS para Classificação de Doenças em Português/Ministério da Saúde/ USP/Organização Pan-Americana da Saúde, 1978. v. 1 .

18. MELLO, C.G. Saúde e assistência médica no Brasil. São Paulo, CEBES/HUCITEC, 1977.

19. MENENDEZ, E.L. La automedicación y los medios de comunicación masiva. Cuad. med. soc., Rosario, (15):33-43, 1981.

20. NITSCHKE, C.A.S.; GUIMARÃES, F.S.; CUNHA, J.; DUTRA, A.C.A.; SILVA Jr., M.M. Estudo sobre uso de medicamentos em quatro bairros de Porto Alegre. Rev. Ass. méd. Rio Grande do Sul, Porto Alegre, 25:184-9, 1981 .

21. ORGANIZACION MUNDIAL DE LA SALUD. Comite de Expertos en Uso de Medicamentos Esenciales, Ginebra, 1984. Segundo informe. Ginebra, 1985. (Serie de Informes Técnicos, 722).

22. PACHECO, M.V.A. A máfia dos remédios. Rio de Janeiro, Ed. Civilização Brasileira 1978. (Coleção Retrato do Brasil).

23. PAUlO, L.G. \& ZANINI, A.C. Necessidade de medicamentos no Brasil: teoria e prática de 1980 1985. Rev. Ass. méd. bras., 31:245-7, 1985.

24. SARAIVA, J.L. Politica Nacional de Medicamen10s. Rev. bras. Educ. med., Rio de Janeiro, 7:167$78,1983$.

25. STOLLEY, P.D.; BECKER, M.H.; MAVILLA, J.D.; LASAGUA, L.; GAINOR, M.; SLOANE, L.M. Drug prescripting and use in an American community. Ann. intern. Med., 76:537-40, 1972.

26. TEMPORÃO, J.G. \& RAMOS, C.L. A propaganda de medicamentos e o mito da saúde. Saúde Deb. (11):33-7, 1981 .

Recebido para publicação em $11 / 2 / 1988$ Reapresentado em $1 / / 8 / 1988$ Aprovado para publicação em 24/8/1988 